\title{
Study on Strengthening Mechanism of Epoxy Resin/Rubber Concrete Interface by Molecular Dynamics Simulation
}

\author{
Lijuan Li, Dajing Qin (D), Zhijun Xu $\mathbb{D}$, and Yong Feng \\ Henan University of Technology, Zhengzhou 450001, China \\ Correspondence should be addressed to Zhijun Xu; xuzhijunhaut@163.com
}

Received 12 December 2021; Accepted 20 December 2021; Published 11 January 2022

Academic Editor: Bing Bai

Copyright (c) 2022 Lijuan Li et al. This is an open access article distributed under the Creative Commons Attribution License, which permits unrestricted use, distribution, and reproduction in any medium, provided the original work is properly cited.

\begin{abstract}
Rubber concrete has high environmental and economic benefits. However, the difference in the physical and chemical properties of the interface causes a weak interface between rubber and concrete, which limits the use of rubber concrete to a certain extent. Based on the macroexperiment of epoxy resin (EP) modified rubber concrete, from the nanoscale level, three interface models of $\mathrm{Rh}$ (natural rubber)/C-S-H, EP/C-S-H, and $\mathrm{Rh} / \mathrm{EP} / \mathrm{C}-\mathrm{S}-\mathrm{H}$ were constructed by molecular dynamics simulation to explore the interaction between epoxy resin and rubber cement-based interface and reveal its microreinforcement mechanism. The results of interaction energy, radial distribution function, and mean square displacement show that the addition of EP not only improves the interface interaction energy between $\mathrm{Rh}$ and $\mathrm{C}-\mathrm{S}-\mathrm{H}$ but also provides a large number of hydrogen bond donors and receptors, promotes the diffusion of $\mathrm{Ca}$, and increases the adhesion between $\mathrm{Rh}$ and cement matrix. The results of the analysis of mechanical properties show that the elastic modulus of the rubber concrete interface model is improved and the interface properties are improved after adding EP.
\end{abstract}

\section{Introduction}

With the continuous development of industries in various countries, the contradiction between the shortage of natural resources and the rapid development of society is becoming increasingly fierce. The low carbon green development and the utilization of waste resources will also become the rigid requirements of social sustainable development. With the rapid development of the global automobile industry and transportation industry, the use of automobile tires has increased sharply, and the output of waste tires is also increasing. However, the waste rubber is not easy to degrade naturally, and direct landfill is easy to cause soil pollution. Incineration will produce a large number of toxic and harmful gases and cause air pollution. Therefore, the disposal of solid waste from waste tires has become a serious global social problem (Figure 1).

Rubber concrete is a kind of special concrete based on ordinary concrete and modified by adding a certain amount of waste rubber particles or rubber powder. Compared with ordinary plain concrete, it can not only improve the mechanical properties of concrete but also solve the problem of difficulty in treatment of waste tires. However, after the waste rubber aggregate is added to the concrete, it will not only affect the cement hydration reaction of the concrete but also form many weak interface transition zones in the new composite material composed of nonpolar rigid bodies and organic elastomers. The weak interface problem usually leads to the early failure of the composite material. This has seriously hindered the development and application of rubber concrete (Figure 2). In addition, epoxy resin (EP) concrete has high strength, short curing time, and good durability [1], but the impact resistance of EP is poor (belonging to brittle material), while rubber has good impact resistance (belonging to elastic material). The modulus of EP adhesive and rubber particles is close, and the adhesion is good, which improves the interfacial adhesion. The modified EP concrete with rubber particles or EP-modified rubber concrete can effectively improve the flexural performance, crack resistance, and impact resistance of concrete. EP rubber elastic concrete combines the advantages of the above two materials and has broad application prospects. 

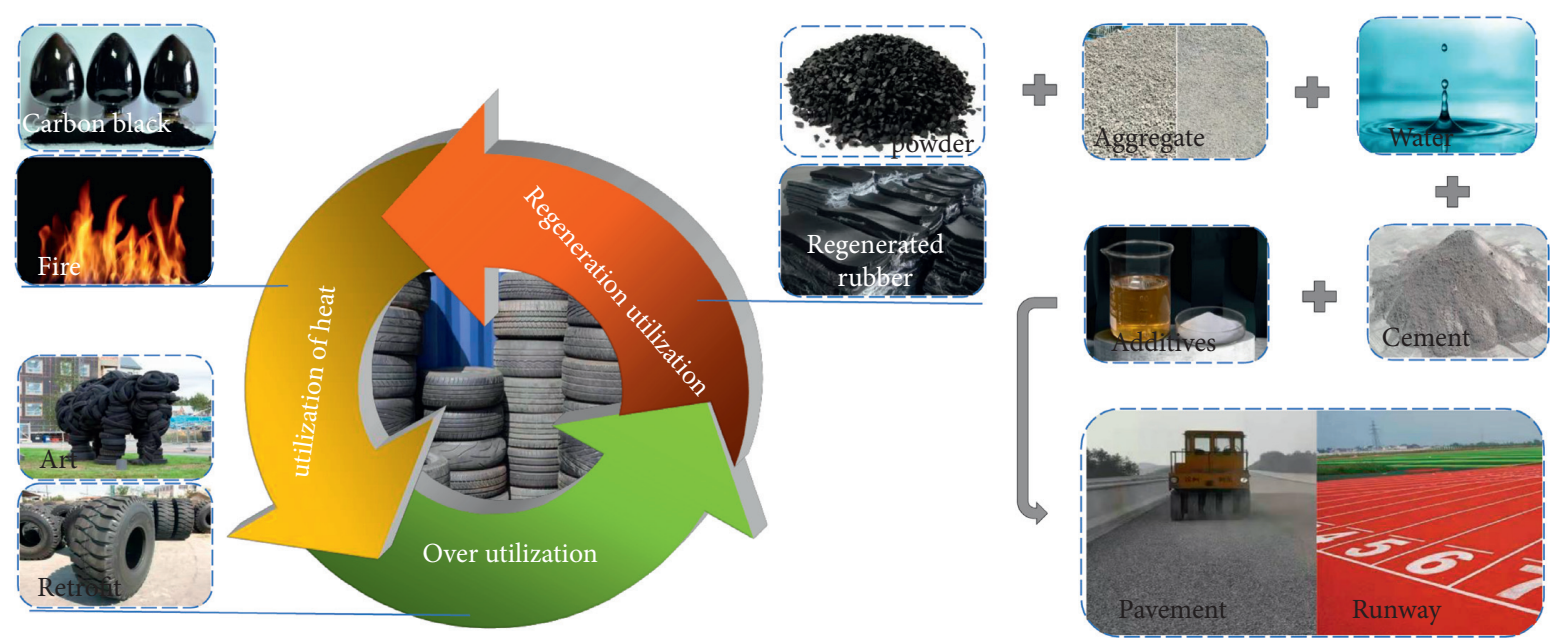

FIGURE 1: Recycling of waste rubber.
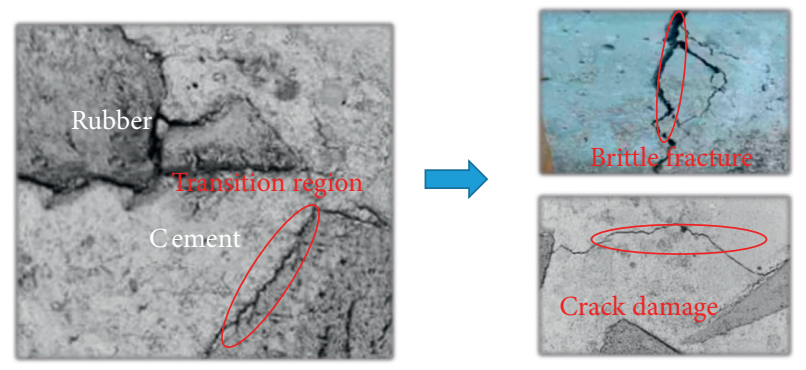

FIGURE 2: Electron microscopic picture of the interface transition zone and macroscopic failure form.

Because the stiffness of rubber is difficult to be significantly improved, improving the bond between rubber and cement matrix is considered to be the key. Therefore, some studies have found that adding modifiers or improving the preparation method of rubber concrete can improve the interfacial adhesion between rubber and cement matrix, so as to improve the mechanical properties of rubber concrete [2-7]. As mentioned above, one of the most successful methods is to add the epoxy resin material into the rubber concrete. The complementarity of the two in physical properties makes the treated rubber particles not only improve the compressive strength and splitting tensile strength of rubber concrete but also enhance the deformation resistance of rubber concrete [8]. Compared with soil, rubber concrete is a mixed structure. Although there are research methods similar to Yuan et al. [9] using a macromodel to explore the mechanical properties of the soil before and after modification, there is no ideal model [10-12] that can directly observe its internal material displacement, which is similar to the transparent soil, and there is no research method similar to the finite element model and physical model that can study its coupling deformation mechanism [13-17]. Although the above research methods can be achieved in the future, however, the traditional research tools cannot explain the influence of epoxy resin on the mechanical properties and micromorphology of rubber concrete on the molecular atomic scale. Therefore, in order to overcome this shortcoming, researchers at home and abroad found that the molecular dynamics simulation (MD) can be used to study the interface interaction and reinforcement mechanism of composite concrete on a nanoscale level, and this atomic research method is popular. Han et al. [18] proposed a new surface treatment method using polyvinyl alcohol (PVA) to improve the hydrophilicity of waste rubber and studied the interfacial strengthening mechanism between PVA and waste rubber/cement slurry through MD. Du et al. [19] used the framework of experimental analysis and MD to study the nanostructure of resin/ cement interface and found that the resin enhanced the adhesion energy and Young's modulus of cement. Although epoxy resin/rubber concrete has been widely used, there is still a lack of theoretical basis and root cause research to support it, which is very important for the follow-up research and development of high-performance epoxy resin/ rubber concrete cement.

In this study, strong polar groups were introduced into the rubber surface to produce a strengthening bond between rubber and the cement matrix, so as to improve the interfacial bonding performance between the rubber and cement matrix and improve the mechanical strength of rubber cement concrete. The interaction energy, radial distribution function, mean square displacement, and mechanical properties were calculated by using the above molecular dynamics calculation method. This study verifies the application effect of EP in rubber concrete composites in macro experiments and reveals its interface behavior, micromechanism, and mechanical properties on the molecular scale. This study provides basic theoretical support for the application of epoxy resin/rubber concrete and has guiding significance for the development of higher quality composite concrete in the future.

\section{Materials and Methods}

2.1. Model Construction. The establishment of a reasonable initial model plays an important role in the success of simulation calculation. As an important part of the research, 
concrete is a kind of composite material that can play its mechanical properties only by the bonding effect of cement. Calcium silicate hydrate (C-S-H) is the most important binding phase in cement-based materials, accounting for $60 \%-70 \%$ of hydration products. Its structure and mechanical properties are equivalent to the "DNA" of cementbased building materials at the molecular level [20-22]. In this study, tobermorite 11 was used to establish the initial structural model. The model was widely applied in the study of the structure and dynamics of the C-S-H gel and was successfully applied to the evolution of C-S-H performance. Then, it is expanded twice along the $X$ direction and triple along the $y$ direction and kept unchanged in the $Z$-direction to form an expanded monomer. In addition, in order to establish the interface phase between tobermorite 11 and EPS, C-S-H was sheared along the $\left[\begin{array}{lll}0 & 0 & 1\end{array}\right]$ direction $(z$-direction) to obtain a parallel C-S-H substrate with a size of $22.5 \AA \times 22.1 \AA$.

The rubber used for rubber concrete mainly comes from waste truck tires and bridge truck tires. Most automobile tire materials are mainly composed of natural rubber or synthetic rubber, but the comprehensive performance of natural rubber is superior to synthetic rubber. The output of natural rubber is low and the demand is large. Its recycling has high social, economic, and environmental benefits. Therefore, in this study, the rubber is simplified to natural rubber. Natural rubber is an elastic solid obtained from the natural latex of rubber tree after a certain process or treatment. Its main component is cis-1,4-polyisoprene. A rubber chain containing 16 monomers was constructed in the polymer module. Then, the $\mathrm{Rh}$ amorphous cells were constructed with an amorphous cell at the specified temperature $(289 \mathrm{~K})$ and at fine precision. In this study, E-51 epoxy resin was selected as the main material of this study, and its molecular structure is shown in Figure 3(a). It contains unique active groups and polar groups. In epoxy resin concrete, epoxy resin is generally used together with curing agents and diluents. In this study, the TEPA fatty amine curing agent and diluent BGE are used, and the cured epoxy resin models are constructed according to the curing reaction principle [23], as shown in Figure 3(b), respectively. Considering that the epoxy resin cannot be completely cured, the amorphous epoxy resin cell is constructed with uncured E51 and cured E51 at $1: 1$ (mass ratio). Finally, the Rh/C-S-H (2196 atoms), EP/CS-H (1829 atoms), and Rh/EP/C-S-H (2873 atoms) interface models (as shown in Figure 3(c)) are constructed with the build layer, and the sizes of each model are $22.5 \AA \times$ $22.1 \AA \times 94.5 \AA$ (model 1), $22.5 \AA \times 22.1 \AA \times 109.3 \AA$ (model 2), and $22.5 \AA \times 22.1 \AA \times 111.9 \AA$ (model 3), respectively. The $Z$-direction is larger than $X$ and $y$ because a $50 \AA$ vacuum layer is added. Its purpose is to make the polymer interact only with one side of the hydrated calcium silicate surface, so as to ensure that it does not interact with the periodic image of the calculation unit. At the same time, the size of the system box along the vertical direction is large enough to fully observe the configuration change of the C-S-H surface polymer.
2.2. Force Field and Simulation Details. Force field is a basic energy function, which is used to calculate the potential energy of the atomic system and simulate the interaction between atoms in the average mode of molecular dynamics simulation. The compass force field is used in this study. The compass force field is the first molecular force field to unify the force field of organic molecular systems and inorganic molecular systems, which can effectively predict the microstructure and dynamic properties of polymer materials and has been widely used in many aspects [24]. In addition, since it can include most of the energy calculations and the potential energy of the interaction between different atoms and molecules in silicic acid materials, and the experiments show that compass is in good agreement with its experimental data, the compass force field is fully applicable to the simulation of cement hydration products $[25,26]$.

The smart method is used to optimize the structure of the composite materials for 10000 steps and 3 times. This process can basically eliminate the local unreasonable structure in the process of model construction and ensure the convergence of the model. Then, the composite system was annealed in the NPT ensemble with a simulation time of 200 ps: it was heated from $200 \mathrm{~K}$ to $800 \mathrm{~K}$ and then cooled to $200 \mathrm{~K}$ for 20 cycles. The conformation with the lowest potential energy after annealing was calculated by molecular dynamics. Its parameters were set as NVT ensemble, compass force field, and temperature of $298 \mathrm{~K}$. The atombased method was used for van der Waals interaction and electrostatic interaction. The cut-off distance is $15.5 \AA$. The Andersen method is used for temperature control and the Berendsen method is used for pressure control. The step size is $1 \mathrm{fs}$ and the time is $500 \mathrm{ps}$. The operation results are output every 50 steps.

\section{Results and Discussion}

3.1. Interaction Energy. According to the energy calculation of the three interface models under the same conditions, the interaction energy reflects the interaction between the inorganic phase and the organic phase [27]. The binding energy is not only an important standard to evaluate the adhesion and miscibility between $\mathrm{C}-\mathrm{S}-\mathrm{H}$ and polymer but also an important index to measure the adsorption strength in the adsorption process, which is contrary to the interaction energy [28, 29]. The greater the absolute value, the stronger the interaction force. The calculation formula of $E_{\text {inter }}$ is shown in the following formula:

$$
\mathbf{E}_{\text {inter }}=\mathbf{E}_{\text {total }}-\left(\mathbf{E}_{\text {surface }}+\mathbf{E}_{\text {polymer }}\right)=-\mathbf{E}_{\text {bind }} \text {, }
$$

where $E_{\text {inter }}$ is the interface interaction energy, $E_{\text {surface }}$ is the energy of C-S-H crystal surface, $E_{\text {polymer }}$ is the total energy of polymer, and $E_{\text {bind }}$ is the binding energy of C-S-H surface and amorphous mixed polymer. The calculation results are shown in Figure 4.

It can be seen from Figure 4 that the energy ratios of $E_{\text {Total }}, E_{\text {Surface }}, E_{\text {Non-bond }}$, and $E_{\text {Coulomb }}$ of the three models are similar. The difference is in the van der Waals effect. It 


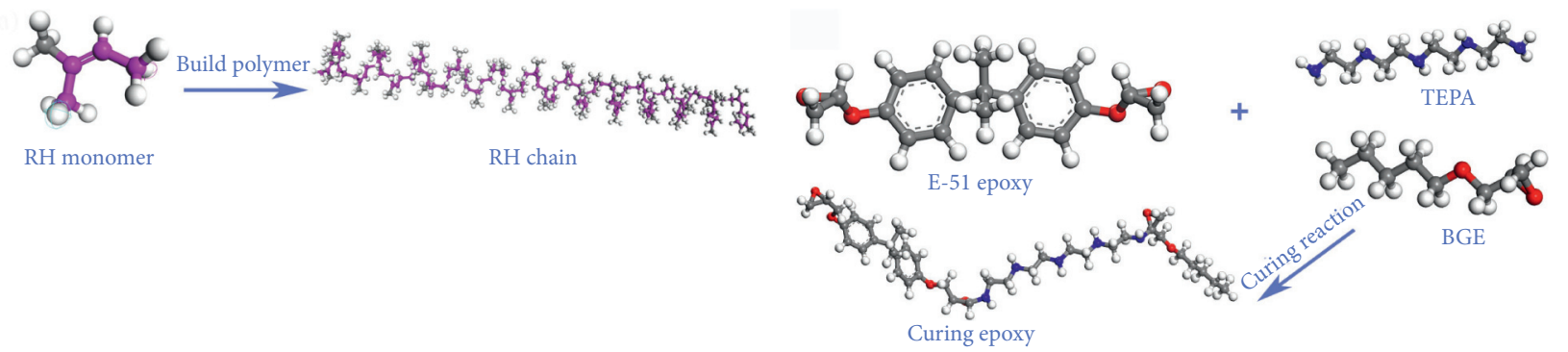

(a)

(b)
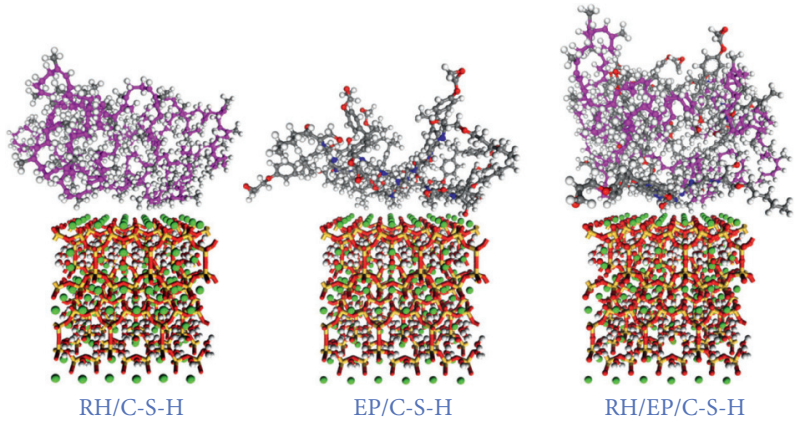

(c)

Figure 3: (a) Construction of Rh, (b) construction of EP, and (c) construction of three interface models (pink: carbon chain; white: H; red: $\mathrm{O}$; blue: $\mathrm{N}$; green: $\mathrm{Ca}$; yellow: $\mathrm{Si}$ ).

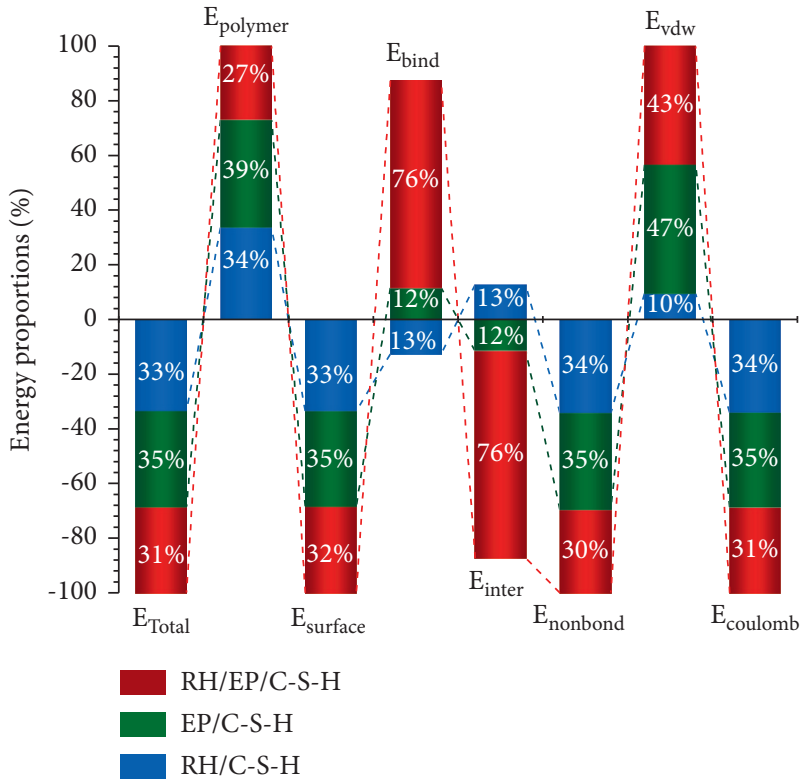

Figure 4: Percentage of energy of three models.

can be seen that van der Waals is the main force controlling the intermolecular interaction. The interaction energy of each system is negative, which shows that the molecules at the interface of models 2 and 3 attract each other and release heat during the combination process. The composite system is a thermodynamically stable system. In addition, the two interface interaction energies $E_{\text {inter }}$ are increased relative to model 1 , indicating that the interaction between components is enhanced and the formed system is stable. It also shows that EP increases the bonding and stability of the $\mathrm{RH}$ concrete interface. The binding energy of $\mathrm{Rh} / \mathrm{C}-\mathrm{S}-\mathrm{H}$ is negative, which is not conducive to the combination of the two interfaces. This phenomenon is consistent with reality, indicating that the additional energy of EP has a strong interaction with C-S-H.

3.2. Radial Distribution Function. To further investigate the interaction between $\mathrm{C}-\mathrm{S}-\mathrm{H}$ and polymer molecules, the equilibrium trajectory of the system is analyzed by the radial distribution function. The radial distribution function $g(r)$ represents the ratio of the probability of finding $B$ particles when the distance from a particle is $r$ to the probability of finding $\mathrm{B}$ particles when they are completely randomly distributed, that is, the ratio of regional density to the average density of $\mathrm{B}$ particles. It can characterize the microstructure information of the system and reflect the interaction between particles [30]. By comparing the $r$-value and peak height in the $g(r)$ diagram, we can identify whether there is an interaction between atoms and their mode and strength. Each model contains hydrated calcium silicate, and many kinds of literature have proved that the calcium in hydrated calcium silicate plays a major role in the adhesion of cement. Next is the hydroxyl in the hydrated calcium silicate. Oxygen can bridge the $\mathrm{Ca}$ ion [31] in the $\mathrm{C}-\mathrm{S}-\mathrm{H}$ channel, form a new calcium silicate gel and fill it into the crack, and enhance the bonding between the inorganic and organic interface. This process has been confirmed in our previous studies [32]. In order to avoid repetition, this study focuses on the interaction between cement-based materials and polymers. In addition, if there is an obvious 
peak of RDF between the two atoms, it indicates that there is a strong spatial correlation between them; that is, there is a stable chemical bond. Intermolecular forces include hydrogen bond and the van der Waals (vdW) action. Generally, the hydrogen bond action range is $2.6-3.1 \AA$, the van der Waals (vdW) action range is $3.1-5.0 \AA$, and the VDW action greater than $5.0 \AA$ is very weak [33].

From the calculation results in Figure 5, we can find that in addition to the chemical bond between $\mathrm{Ca}$ and atoms in the polymer $\left(\mathrm{Ca}-\mathrm{H}_{\mathrm{r}}, \mathrm{Ca}-\mathrm{N}, \mathrm{Ca}-\mathrm{H}_{\mathrm{e}}, \mathrm{Ca}-\mathrm{C}_{\mathrm{e}}\right.$, and $\left.\mathrm{Ca}-\mathrm{C}_{\mathrm{r}}\right)$, the $\mathrm{H}$ bond is also another important chemical bond between cement-based materials and polymer. In particular, the interaction between $\mathrm{N}, \mathrm{O}$, and $\mathrm{Ca}$ in epoxy resin and the hydrogen bonding between $\mathrm{H}$ and $\mathrm{O}$ in water molecules and $\mathrm{Rh}$ and $\mathrm{H}$ and $\mathrm{O}$ in EP $\left(\mathrm{H}_{\mathrm{h}}-\mathrm{H}_{\mathrm{e}}, \mathrm{H}_{\mathrm{h}}-\mathrm{H}_{\mathrm{r}}, \mathrm{O}_{\mathrm{h}}-\mathrm{H}_{\mathrm{r}}\right.$, and $\left.\mathrm{O}_{\mathrm{h}}-\mathrm{H}_{\mathrm{e}}\right)$ greatly increase the interface binding energy [34]. In addition, the peak height of model 2 is not only higher than that of model 1 but also the position of the peak is higher. The peak value of Ca-N $(x=2.25, y=17.1258)$ in model 3 is higher than that of model $2(x=2.25, y=9.842)$, which further proves that the combination of epoxy resin and hydrated calcium silicate is better, and the comprehensive application of epoxy resin and rubber increases its interface interaction. It shows that the addition of EP provides hydrogen bond donors and receptors to the interface. The hydrogen bond is a strong intermolecular interaction, which verifies the reason for the enhancement of the above interaction energy.

3.3. Mean Square Displacement. The previous analysis of interaction energy and radial distribution has revealed the change of chemical bond type and stability after the addition of EP, and it can be seen that the binding energy mainly depends on the interaction between $\mathrm{Ca}$ in tobermorite 11 and amorphous polymer. In order to characterize the dynamic behavior, only the EP and the difference before and after EP is added to RH concrete. The mean square displacement (MSD) of the $\mathrm{Ca}$ atom corresponding to the model is calculated to reflect the diffusion of Ca. The MSD can characterize the diffusion behavior of molecules and is an effective method to study molecular diffusion. The MSD is defined as the square of the difference between atomic displacement and the average of all other atomic displacements [35], which can be obtained from the following equation:

$$
\left\langle r^{2}(t)\right\rangle=\frac{1}{N} \sum_{i=1}^{N}\left\langle\left|r_{i}(t)-r_{i}(0)\right|^{2}\right\rangle,
$$

where $r_{i}(t)$ represents the position of $i$ atoms at time $t, r_{i}(0)$ is the original position of $i$ atoms, and MSD considers threedimensional coordinates. The larger MSD value at time $t$ indicates that the atom diffuses rapidly and is far away from the original position.

As shown in Figure 6, the greater the slope of the MSD curve, the greater the fluidity of particles. After the addition of $\mathrm{RH}$ and $\mathrm{EP}$, the $\mathrm{Ca}$ atom interface model diffuses continuously with time, and the diffusion becomes more intense after they are added together. This result shows that the adsorption capacity of $\mathrm{Ca}$ atoms on the substrate surface is weakened, resulting in the reduction of local constraints on Ca atoms. $\mathrm{C}_{\mathrm{r}}, \mathrm{H}_{\mathrm{r}}, \mathrm{N}$, and $\mathrm{O}_{\mathrm{e}}$ of $\mathrm{EP}$ and $\mathrm{Rh}$ attract $\mathrm{Ca}$ atoms, resulting in the decline of the ability of $\mathrm{C}-\mathrm{S}-\mathrm{H}$ to capture the $\mathrm{Ca}$ atoms. At the same time, the interface model on C-S-H increases the diffusion coefficient of the $\mathrm{Ca}$ atom on its surface more than twice from Rh to EP and then to Rh/EP, indicating that the joint application of $\mathrm{RH}$ and $\mathrm{EP}$ is conducive to the diffusion of $\mathrm{Ca}$ on the aggregate and can effectively accelerate the bonding between hydrated calcium silicate and polymer. In addition, the calculation result is the embodiment of the Ca radial distribution function, and it is also a reason for the strong interaction energy between models 2 and 3.

3.4. Mechanical Properties. Mechanical properties refer to the mechanical characteristics of materials under various external loads in different environments. Young's modulus, shear modulus, and bulk modulus are the main parameters of mechanical properties. Young's modulus $(E)$ is an index to evaluate the rigidity of materials and a measure of the ability of materials to resist elastic deformation. The greater the shear modulus $(g)$, the higher the hardness and yield strength of the material, which are the measures of the ability of the material to resist plastic deformation. The greater the bulk modulus $(k)$, the greater the breaking strength of the material $[36,37]$. The static elastic mechanical properties of models 1,2 , and 3 in the equilibrium state after optimized annealing are analyzed by Ms, and the elastic coefficient matrix of the model can be obtained. It is known that the model is not an extreme heteromorphic material and can be simplified to isotropic material. For isotropic materials, there are only two independent elastic constants in the calculation principle, which are obtained by elastic matrix and two lame coefficients $\lambda$ and $\mu$. Finally, each modulus can be obtained from formulas (3), (4), and (5) $[38,39]$ :

$$
\begin{aligned}
& E=\mu \frac{3 \lambda+2 \mu}{\lambda+\mu}, \\
& G=\mu, \\
& K=\lambda+\frac{2}{3} \mu,
\end{aligned}
$$

where $E$ is Young's modulus, $G$ is the shear modulus, and $K$ is the bulk modulus. The calculated mechanical property parameters are shown in Figure 7.

From the calculation results, it can be found that the mechanical property parameters of models 2 and 3 are slightly larger than model 1 , and model 3 is lower than model 2 , mainly because models 2 and 3 contain epoxy resin, and model 3 contains rubber. As mentioned earlier, $E, K$, and $G$ are measures of material rigidity. The greater their value, the stronger the material rigidity [40]. The calculated results are consistent with previous experiments. Although model 3 does not exceed the mechanical properties of model 2, the combination of the two improves the mechanical properties 


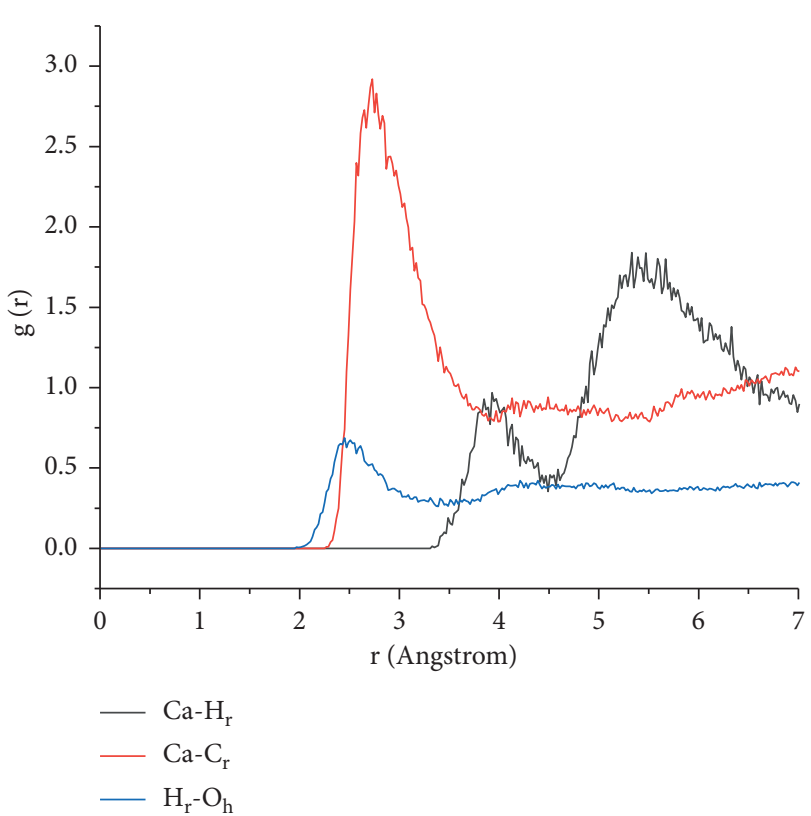

(a)

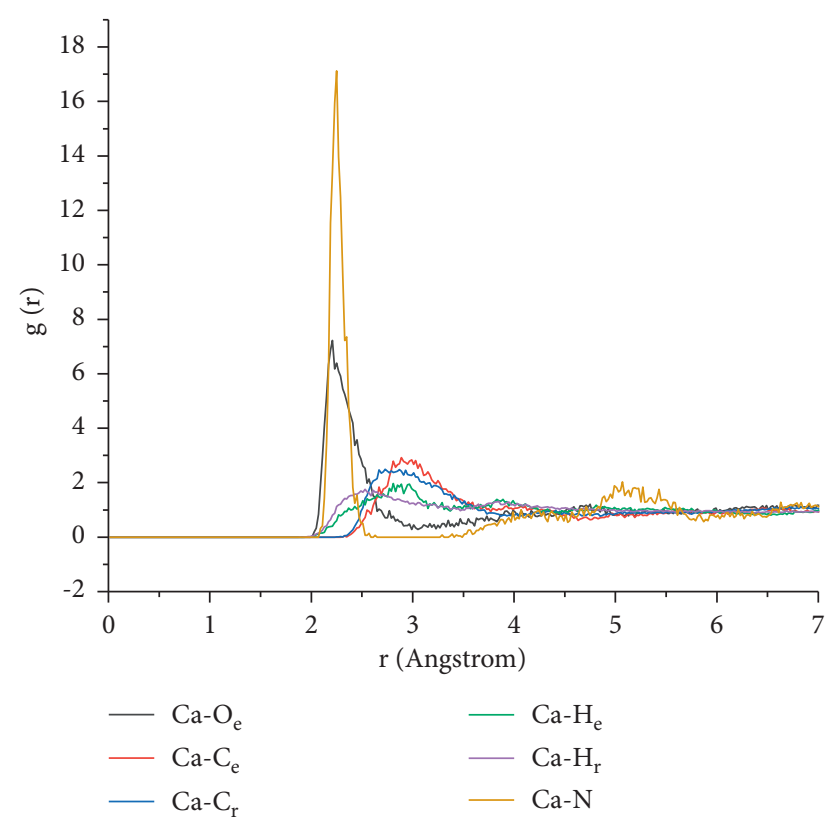

(c)

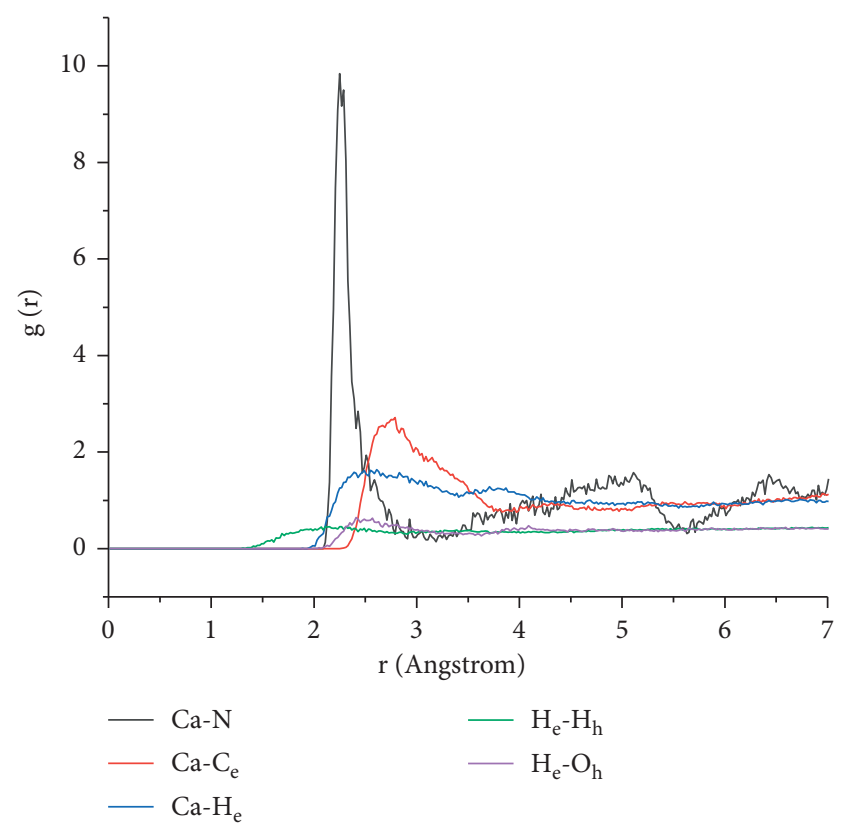

(b)

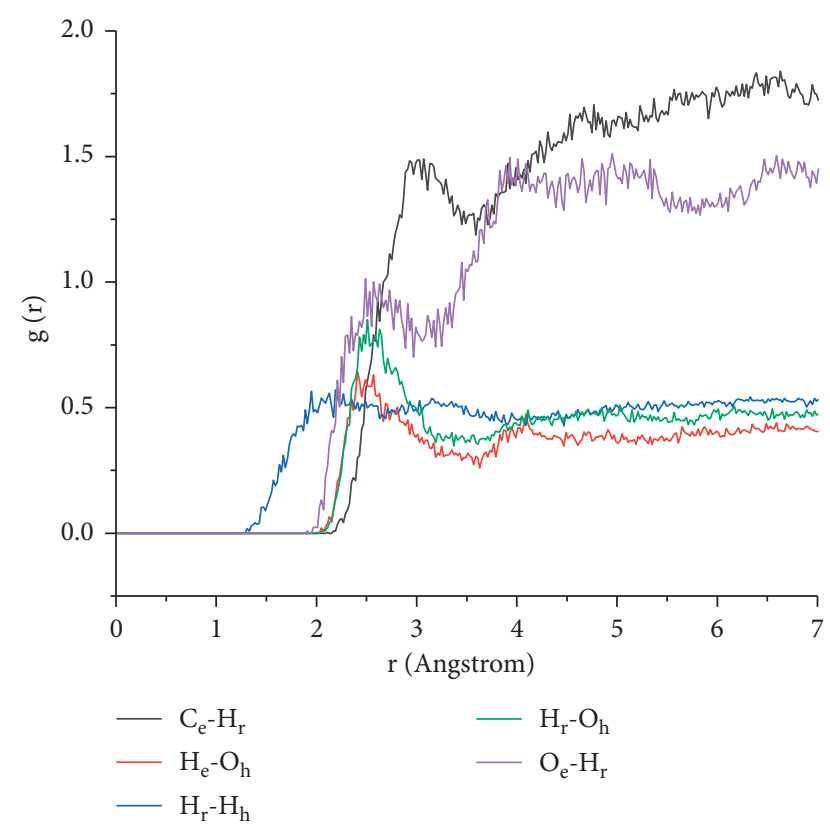

(d)

Figure 5: RDF of C-S-H and atoms in the polymers (a) RH/C-S-H RDF, (b) EP/C-S-H RDF, and (c) RH/EP/C-S-H RDF. 


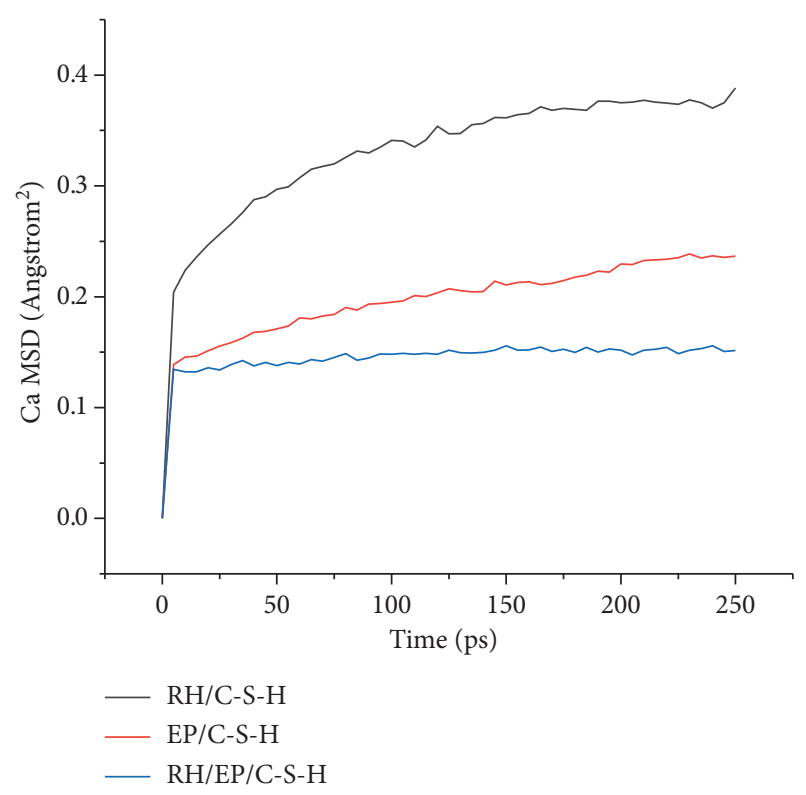

Figure 6: Mean square displacement of three models of Ca.

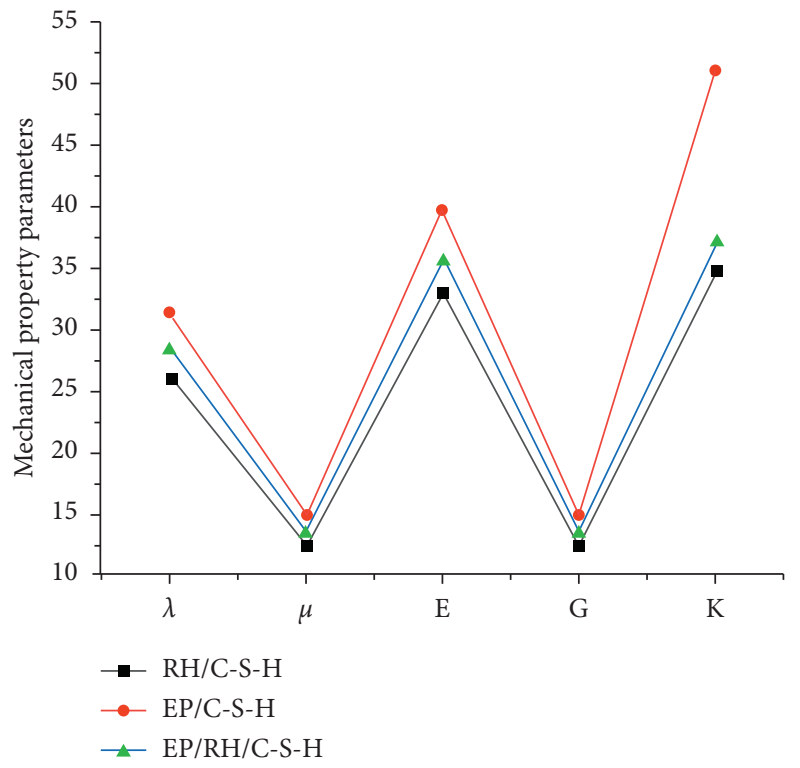

FIGURE 7: Main mechanical property parameters of three models.

of model 1, reduces the consumption of epoxy resin, achieves the purpose of energy conservation and environmental protection, provides basic theoretical support for the application of modified rubber concrete, and has reference significance for the development of better EPS concrete in the future.

\section{Conclusion}

In this study, three interface models were constructed by the molecular dynamics simulation method, and their interaction energy, radial distribution function, MSD, and mechanical properties were calculated. The following conclusions were obtained:
(1) The interaction energy shows that EP increases the bonding and stability of the $\mathrm{RH}$ concrete interface. In addition, the energy ratio of other energy is basically the same except for the VdW force. It can be explained that van der Waals force is the main reason for the difference of action energy among the three.

(2) Radial distribution function analysis shows that there is a strong interaction between $\mathrm{N}$ atoms in models 2 and 3 and $\mathrm{Ca}$ atoms in $\mathrm{C}-\mathrm{S}-\mathrm{H}$. In addition, the addition of EP provides a large number of hydrogen bond donors and receptors for the $\mathrm{Rh} / \mathrm{C}-\mathrm{S}-\mathrm{H}$ interface interaction, which enhances the interface interaction between models 2 and 3 .

(3) The mean square displacement shows that the joint application of EP and RH is conducive to the diffusion of calcium on the aggregate and can effectively accelerate the combination of hydrated calcium silicate and polymer.

(4) In the calculation results of static mechanical properties, it is found that the addition of EP obviously increases Young's modulus $(E)$, bulk modulus $(k)$, and shear modulus $(G)$ of the interface model, which indicates that its rigidity is enhanced and the interface properties are improved, which not only retains the excellent characteristics of rubber concrete but also enhances its interface mechanical properties.

\section{Data Availability}

The data used to support the findings of this study are available from the corresponding author upon request.

\section{Conflicts of Interest}

The authors declare that they have no conflicts of interest regarding the publication of this paper.

\section{Acknowledgments}

This study was supported by the National Natural Science Foundation of China (51978247), the Innovative Funds Plan of Henan University of Technology (2020ZKCJ21), and Zhengzhou Collaborative Innovation Project (21ZZXTCX09).

\section{References}

[1] R.-M. Diaconescu, M. Barbuta, and M. Harja, "Prediction of properties of polymer concrete composite with tire rubber using neural networks," Materials Science and Engineering: $B$, vol. 178, no. 19, pp. 1259-1267, 2013.

[2] N. S. Joekes, "Use of tire rubber particles as addition to cement paste," Cement and Concrete Research, vol. 30, no. 9, pp. 1421-1425, 2000.

[3] B. S. Mohammed, A. B. Awang, S. S. Wong, and C. P. Nhavene, "Properties of nano silica modified rubbercrete," Journal of Cleaner Production, vol. 119, pp. 66-75, 2016. 
[4] G. Li, Z. Wang, C. K. Y. Leung et al., "Properties of rubberized concrete modified by using silane coupling agent and carboxylated SBR," Journal of Cleaner Production, vol. 112, pp. 797-807, 2016.

[5] F. Xu, C. Peng, J. Zhu, and J. Chen, "Design and evaluation of polyester fiber and SBR latex compound-modified perlite mortar with rubber powder," Construction and Building Materials, vol. 127, pp. 751-761, 2016.

[6] K. B. Najim and M. R. Hall, "Crumb rubber aggregate coatings/pre-treatments and their effects on interfacial bonding, air entrapment and fracture toughness in selfcompacting rubberised concrete (SCRC)," Materials and Structures, vol. 46, no. 12, pp. 2029-2043, 2013.

[7] E. Ganjian, M. Khorami, and A. A. Maghsoudi, "Scrap-tyrerubber replacement for aggregate and filler in concrete," Construction and Building Materials, vol. 23, no. 5, pp. 1828-1836, 2009.

[8] S. Wang, "Influence of epoxy resin adhesive on elastic concrete properties," Chinese adhesive, vol. 25, no. 3, pp. 9-12, 2016.

[9] B. Yuan, Z. Li, Y. Chen et al., "Mechanical and microstructural properties of recycling granite residual soil reinforced with glass fiber and liquid-modified polyvinyl alcohol polymer," Chemosphere, vol. 268, Article ID 131652, 2021.

[10] B. Yuan, Z. Li, Z. Zhao, H. Ni, Z. Su, and Z. Li, "Experimental study of displacement field of layered soils surrounding laterally loaded pile based on transparent Soil," Journal of Soils and Sediments, vol. 21, no. 9, pp. 3072-3083, 2021.

[11] B. Yuan, M. Sun, L. Xiong, Q. Luo, S. Pradhan, and H. Li, "Investigation of 3D deformation of transparent soil around a laterally loaded pile based on a hydraulic gradient model test," Journal of Building Engineering, vol. 28, no. 3, Article ID 1010124, 2020.

[12] B. Yuan, M. Sun, Y. Wang, L. Zhai, Q. Luo, and X. Zhang, "Full 3D displacement measuring system for 3D displacement field of soil around a laterally loaded pile in transparent soil," International Journal of Geomechanics, vol. 19, no. 5, Article ID 04019028, 2019.

[13] B. Yuan, Z. Li, Z. Su, Q. Luo, M. Chen, and Z. Zhao, "Sensitivity of multistage fill slope based on finite element model," Advances in Civil Engineering, vol. 2021, Article ID 6622936, 13 pages, 2021.

[14] B. Bai, Q. Nie, Y. Zhang, and X. Wang, "HuCotransport of heavy metals and $\mathrm{SiO} 2$ particles at different temperatures by seepage," Journal of Hydrology, vol. 597, Article ID 125771, 2021.

[15] B. Bai, G. Yang, T. Li, and G. Yang, "A thermodynamic constitutive model with temperature effect based on particle rearrangement for geomaterials," Mechanics of Materials, vol. 139, Article ID 103180, 2019.

[16] X. Que, Z. Zhu, Z. Niu, and W. Lu, "Estimating the strength and deformation of columnar jointed rock mass based on physical model test," Bulletin of Engineering Geology and the Environment, vol. 80, pp. 1557-1570, 2020.

[17] B. Bai, R. Zhou, G. Cai, W. Hu, and G. Yang, "Coupled thermo-hydro-mechanical mechanism in view of the soil particle rearrangement of granular thermodynamics," Computers and Geotechnics, vol. 137, no. 8, Article ID 104272, 2021.

[18] Q. Han, Y. Yang, J. Zhang et al., "Insights into the interfacial strengthening mechanism of waste rubber/cement paste using polyvinyl alcohol: experimental and molecular dynamics study," Cement and Concrete Composites, vol. 114, Article ID 103791, 2020.
[19] J. Du, Y. Bu, and Z. Shen, "Interfacial properties and nanostructural characteristics of epoxy resin in cement matrix," Construction and Building Materials, vol. 164, pp. 103-112, 2018.

[20] S. Jin, J. Li, W. Xu, and Q. Ding, "Heterogeneous nature of calcium silicate hydrate (C-S-H) gel: a molecular dynamics study," Journal of Wuhan University of Technology-Materials Science Edition, vol. 35, no. 2, pp. 435-440, 2020.

[21] A. Kumar, B. J. Walder, and A. K. Mohamed, "The atomiclevel structure of cementitious calcium silicate hydrate," Journal of Physical Chemistry C, vol. 121, no. 32, 2017.

[22] D. Fan and S. Yang, "Mechanical properties of C-S-H globules and interfaces by molecular dynamics simulation," Construction and Building Materials, vol. 176, pp. 573-582, 2018.

[23] S. K. Ooi, W. D. Cook, G. P. Simon, and C. H. Such, "DSC studies of the curing mechanisms and kinetics of DGEBA using imidazole curing agents," Polymer, vol. 41, no. 10, pp. 3639-3649, 2000.

[24] C. Yu, K. Hu, Q. Yang, and J. Chen, "Multi-scale observation of oxidative aging on the enhancement of high-temperature property of SBS-modified asphalt," Construction and Building Materials, vol. 313, Article ID 125478, 2021.

[25] A. Al-Ostaz, W. Wu, H. D. Cheng, and C. R. Song, "A molecular dynamics and microporomechanics study on the mechanical properties of major constituents of hydrated cement," Composites Part B, vol. 41, no. 7, pp. 543-549, 2010.

[26] S. Huang, "Study on mechanical properties of hydrated calcium silicate by molecular dynamics simulation," Silicate Bulletin, vol. 37, pp. 1687-1692, 2018.

[27] R. Toth, A. Coslanich, M. Ferrone et al., "Computer simulation of polypropylene/organoclay nanocomposites: characterization of atomic scale structure and prediction of binding energy," Polymer, vol. 45, no. 23, pp. 8075-8083, 2004.

[28] H. Wang, X. Lu, Y. Leng et al., "Molecular dynamics simulations on the interaction between polymers and hydroxyapatite with and without coupling agents," Acta Biomaterialia, vol. 5, no. 4, pp. 1169-1181, 2009.

[29] J. Xiao, H. Huang, J. Li, H. Zhang, Z. Wei, and H. Xiao, "Computation of interface interactions and mechanical properties of HMX-based PBX with Estane 5703 from atomic simulation," Journal of Materials Science, vol. 43, no. 17, pp. 5685-5691, 2008.

[30] B. G. Levine, J. E. Stone, and A. Kohlmeyer, "Fast analysis of molecular dynamics trajectories with graphics processing units-radial distribution function histogramming," Journal of Computational Physics, vol. 230, no. 9, pp. 3556-3569, 2011.

[31] P. Wang, G. Qiao, Y. Zhang et al., "Molecular dynamics simulation study on interfacial shear strength between calcium-silicate-hydrate and polymer fibers," Construction and Building Materials, vol. 257, Article ID 119557, 2020.

[32] Y. Feng, D. Qin, L. Li, Y. Li, C. Wang, and P. Wang, "EVA enhances the interfacial strength of EPS concrete: a molecular dynamics study," Journal of Experimental Nanoscience, vol. 16, no. 1, pp. 383-397, 2021.

[33] G. D. Merfeld, A. Karim, B. Majumdar, S. K. Satija, and D. R. Paul, "Interfacial thickness in bilayers of poly (phenylene oxide) and styrenic copolymers," Journal of Polymer Science Part B: Polymer Physics, vol. 36, no. 17, pp. 3115-3125, 2000.

[34] D. Hou, J. Zhang, Z. Li, and Y. Zhu, "Uniaxial tension study of calcium silicate hydrate (C-S-H): structure, dynamics and mechanical properties," Materials and Structures, vol. 48, no. 11, pp. 3811-3824, 2015. 
[35] C. Yu, K. Hu, G. Chen, R. Chang, and Y. Wang, "Molecular dynamics simulation and microscopic observation of compatibility and interphase of composited polymer modified asphalt with carbon nanotubes," Journal of Zhejiang University-Science, vol. 22, no. 7, pp. 528-546, 2021.

[36] Q. Yuan, L. Wan, X. Shi et al., "Interface interaction and mechanical properties simulation of HA-Cs composite," Journal of Shenzhen University (Science and Technology edition), vol. 35, pp. 83-90, 2018.

[37] T. Sun, J. Xiao, F. Zhao, and H. Xiao, "Study on the compatibility, interface interaction and mechanical properties of Cl-20 /DNB eutectic PBXs," Energetic Materials, vol. 23, no. 4, pp. 309-314, 2015.

[38] L. Qiu and H. Xiao, "Molecular dynamics study of binding energies, mechanical properties, and detonation performances of bicyclo-HMX-based PBXs-ScienceDirect," Journal of Hazardous Materials, vol. 164, no. 1, pp. 329-336, 2009.

[39] Z. Wei, J. Xiao, W. Zhu, and H. Xiao, "Molecular dynamics simulations of RDX and RDX-based plastic-bonded explosives," Journal of Hazardous Materials, vol. 164, no. 2-3, pp. 1082-1088, 2009.

[40] R. Li, Q. Wei, X. Wang, and S. Long, "Molecular dynamics simulation of mechanical properties of alumina materials," Tool Technology, vol. 64 pages, 2014. 